\title{
A Modified Heat Leak Test Facility Employing a Closed-Cycle Helium Refrigerator
}

\author{
William N. Boroski \\ Fermi National Accelerator Laboratory \\ P.O. Box 500, Batavia, Illinois 60510
}

January 1996

Proceediongs of the 15th International Cryogenic Engineering Conference, Genova, Italy, June 7-10, 1994. 


\section{Disclaimer}

This report was prepared as an account of work sponsored by an agency of the United States Government. Neither the United States Government nor any agency thereof, nor any of their employees, makes any warranty, express or implied, or assumes any legal liability or responsibility for the accuracy, completeness, or usefulness of any information, apparatus, product, or process disclosed, or represents that its use would not infringe privately owned rights. Reference herein to any specific commercial product, process, or service by trade name, trademark, manufacturer, or otherwise, does not necessarily constitute or imply its endorsement, recommendation, or favoring by the United States Government or any agency thereof. The views and opinions of authors expressed herein do not necessarily state or reflect those of the United States Government or any agency thereof. 
"Presented at the 15 th International Cryogenic Engineering Conference, Genova, Italy, June 7-10, 1994"

A Modified Heat Leak Test Facility Employing a Closed-cycle Helium Refrigerator

William N, Boroski

Fermi National Accelerator Laboratory, P.0. Box 500, Batavia, IL, USA, 60510

\begin{abstract}
A Heat Leak Test Facility (HLTF) has been in use at Fermilab for many years. The apparatus has successfully measured the thermal performance of a variety of cryostat components under simulated operating conditions. While an effective tool in the cryostat design process, the HLTF has several limitations. Temperatures are normally fixed at cryogen boiling points and run times are limited to cryogen inventory. Moreover, close personnel attention is required to maintain system inventories and sustain system equilibrium. To provide longer measurement periods without perturbation and to minimize personnel interaction, a new heat leak measurement facility (HLTF-2) has been designed that incorporates a closed-cycle helium refrigerator. The two-stage refrigerator provides cooling to the various temperature stations of the HLTF while eliminating the need for cryogens. Eliminating cryogen inventories has resulted in a reduction of the amount of direct personnel attention required.
\end{abstract}

\title{
INTRODUCTION
}

Fermilab's Engineering Laboratory has played an active role in the development of various components used in advanced cryostat designs. Development efforts typically involve the fabrication, instrumentation, and testing of prototype devices, or test samples. Test samples are fabricated in a manner to simulate the end product. During evaluation, samples are subjected to conditions closely approaching those encountered during actual use. A key resource for conducting thermal testing in the Engineering Laboratory has been a Heat Leak Test Facility (HLTF) [1,2]. The HLTF is a cryostat consisting of three cryogen storage reservoirs, test sample mounting flanges, a heatmeter for measuring heat leak, and other supporting instrumentation. While the HLTF has been used extensively to quantify the thermal performance of various cryostat support structures and multilayer insulation (MLI) systems [3,4], it is recognized that the system has limitations. Cooling for the temperature stations in the HLTF is achieved via connection to one of the three cryogen storage vessels. Therefore, run times are typically limited by cryogen inventory. System equilibrium is disturbed each time a vessel requires re-filling, which ultimately extends overall measurement run time. Personnel interaction is also required during filling operations as these operations involve the transferring of cryogens from storage containers into the HLTF reservoirs. Since the length of time cryogen inventory is maintained in the HLTF is a function of many system conditions, refills are quite frequently required during off-hours or on weekends. These offhours operations are an inconvenience to persomel and work schedules.

A new Heat Leak Test Facility (HLTF-2) has been designed to address the limitations of the existing system. By incorporating a closed-cycle helium refrigerator, cryogen storage vessels have been eliminated. Not only does this result in extended run times without system perturbations, it increases the safety factor of the operation through the elimination of cryogen-handling activities. The two-stage refrigerator unit provides cooling to the various temperature stations in the system. 
The HLTF-2, illustrated in Figure 1, is presently configured for MLI measurements. The warm boundary can be fixed at temperatures near $300 \mathrm{~K}$ while the cold boundary can be fixed at any temperature between $15 \mathrm{~K}$ and the warm boundary temperature. The system is quite simplistic with only a minimal number of critical components. Centered around the closed-cycle, two stage Gifford McMahon helium refrigerator, supporting components either accommodate the test sample or provide the necessary support to ensure accurate measurement results.

The refrigerator unit serves as the nucleus of the HLTF-2 and is mounted in a vertical position. This allows the refrigerator to support the test sample in addition to providing cooling for the various temperature stations. The present configuration applies a compressive load of approximately $110 \mathrm{~kg}$ to the refrigerator head. Discussions with the manufacturer have disclosed that the refrigerator head can easily support compressive loads of this order.

The HLTF-2 outer radiation shield is mounted to the first stage of the twostage refrigerator through a bolted connection. The first stage operates at a nominal temperature of $50 \mathrm{~K}$ under no-load conditions. Shield temperature can be varied through use of a cryogenic temperature controller. Indium ribbon is installed between the first stage flange and outer shield to ensure good thermal communication. The function of the HLTF-2 outer shield is to intercept radiant heat from the vacuum vessel to the refrigerator second stage. To ensure the outer shield functions effectively, aluminum tape is applied to all shield surfaces. Additionally, a 32-layer MLI blanket is installed on the outer surface of the shield to further minimize radiant heat in-leak.

Attached to the second stage of the refrigerator are several components: the radiation guard, the heatmeter anchor flange, the heatmeter, and the Cold Plate. For MLI measurements, the principle of operation is for heat to flow from the Hot Plate through the MLI test sample into the Cold Plate. Heat is then conducted through the heatmeter into the anchor flange attached to the refrigerator second stage. Under most operating situations, this flange is the coldest component of the HLTF-2. Both the radiation guard and anchor flange are attached to the refrigerator second stage through a bolted connection. Again, indium ribbon is used to ensure good thermal contact. The purpose of the anchor flange is to establish the temperature at the bottom of the heatmeter. Temperature stability and variability is established through means of a resistance heater connected to a high precision cryogenic temperature controller. The heatmeter is attached to the anchor flange through a threaded connection; copper-laden vacuum grease is used to ensure good thermal contact between the heatmeter bottom and the flange.

The heatmeter measures heat flow in real time as a temperature difference across a calibrated thermal impedance [5]. The heatmeter consists of ofHC copper threaded ends separated by a stainless steel disc. Pairs of carbon and platinum resistance thermometers positioned on either side of the disc allow measurement of the temperature difference across the heatmeter, which is proportional to the amount of heat flowing through the device. As the thermal conductivity of the stainless steel disc changes with temperature, the heatmeter is calibrated at each temperature range over which measurements are desired.

A cylindrical drum of OFHC copper is mounted on the top of the heatmeter, also through a threaded connection. This drum, called the Cold Plate, provides the surface around which an MLI test sample is installed. The outer surface, or measurement area, of the Cold Plate is $0.5 \mathrm{~m}^{2}$ and is painted with solar absorbing paint to establish a high emissivity surface. The MLI sample is installed on the top and around the outer circumferential surface of the Cold Plate and as such is positioned to intercept heat transfer from the Hot Plate to the Cold Plate. To address the issue of erroneous heat transfer into and out of the underside of the Cold Plate, a radiation guard is employed.

The radiation guard consists of two OFHC copper discs joined by an ofHC copper cylinder. The guard assembly is covered with aluminum tape to minimize thermal communication with surrounding components. To further minimize communication, both sides of the guard are covered with 32 -layer MLI blankets. As the radiation guard is attached near the heatmeter bottom, it operates at a temperature very near that of the heatmeter bottom. As the Cold Plate is attached to the heatmeter top, its temperature is near that of the heatmeter top. Past operating experience has shown 
that the temperature difference between heatmeter top and bottom is at most several Kelvin during measurement periods. This small temperature difference results in the radiation guard operating at approximately the same temperature of the Cold Plate, resulting in negligible thermal communication between the two surfaces.

To verify that thermal communication between surfaces is negligible and that the heatmeter is not being short-circuited, a calibration heater is incorporated into the cold Plate. With the system in equilibrium, a known electrical power can be applied to the heater. The additional energy raises the temperature of the Cold Plate which causes a corresponding increase in the temperature difference across the heatmeter. Using the heatmeter calibration tables, the corresponding temperature increase is correlated to an increase in heat flow. If the additional heat flowing through the heat meter is equivalent to the electrical power added, it is confirmed that the heatmeter is sensing all heat emanating from the Cold Plate. This verification method has been employed in the HLTF under a wide variety of operating conditions, including various temperature and insulating vacuum ranges. In al1 cases, the amount of electrical power applied has been measured as an equivalent increase in heat leak as indicated by the heatmeter.

In addition to varying the cold boundary temperature, it is often desirable to vary the warm boundary temperature during MLI measurements. This capability is provided by a thin-walled OFHC copper cylinder that surrounds the Cold Plate assembly. Named the Hot Plate, the liner is positioned off the wall of the vacuum vessel with G-10 stand-offs. The inner surface of the Hot Plate is painted with black solar absorbing paint to provide a high emissivity surface. The outer surface is spirally-grooved across the entire assembly. A resistance heater wire is varnished into the groove to provide uniform heating across the entire Hot Plate surface. Hot Plate temperature is established and maintained by a cryogenic temperature controller, with platinum RTD's used as control and sensing thermometers. The instrumented outer surface of the Hot Plate is covered with three sheets of double-aluminized Mylar to reduce thermal communication between the Hot Plate and vacuum vessel wall.

\section{CONCLUSION}

A new facility has been designed and constructed to measure the thermal performance of cryogenic components under simulated operating conditions. Presently configured for MLI measurements, the system is designed to easily accommodate many test setups such as those for support structure performance studies and thermal conductivity measurements. The new system incorporates a closed-cycle, two stage Gifford-McMahon helium refrigerator in place of cryogen storage vessels. The result is a system capable of longer measurement run times without the periodic system perturbations seen in the existing system. In addition to increased run times, the new system is inherently safer as operations involving the handling, transferring, and storing of liquid cryogens have been eliminated. Furthermore, the elimination of cryogens and associated issues will result in a significant reduction in personnel interaction and demand. The system is currently configured with the first MLI test sample installed and ready for initial cooldown.

\section{REFERENCES}

1. Gonczy, J.D., et al., Heat leak measurement facility, In: Advances in Cryogenic Engineering - 31, Plenum Press, New York (1986) 1291-1298.

2. Boroski, W.N., et al., Design and calibration of a test facility for MLI thermal performance measurements below $80 \mathrm{~K}$, In: Advances in Cryogenic Engineering - 37A, Plenum Press, New York, (1991) 275-283.

3. Gonczy, J.D., et al., Thermal performance of a graphite tube compact cryogenic support for the Superconducting Super Collider, In: International Cryogenic Engineering Conference - 12, Butterworths, Guildford, UK (1988). 
4. Boroski, W.N., Nicol, T.N., and Schoo, C.J., Thermal performance of various multilayer insulation systems below 80K, In: Supercollider 4, Plenum Press, New York (1992) 235-242.

5. Kuchnir, M., Gonczy, J.D, and Tague, J.L., Measuring heat leak with a heatmeter, In: Advances in Cryogenic Engineering - 31, Plenum Press, New York (1986) $1285-1290$.

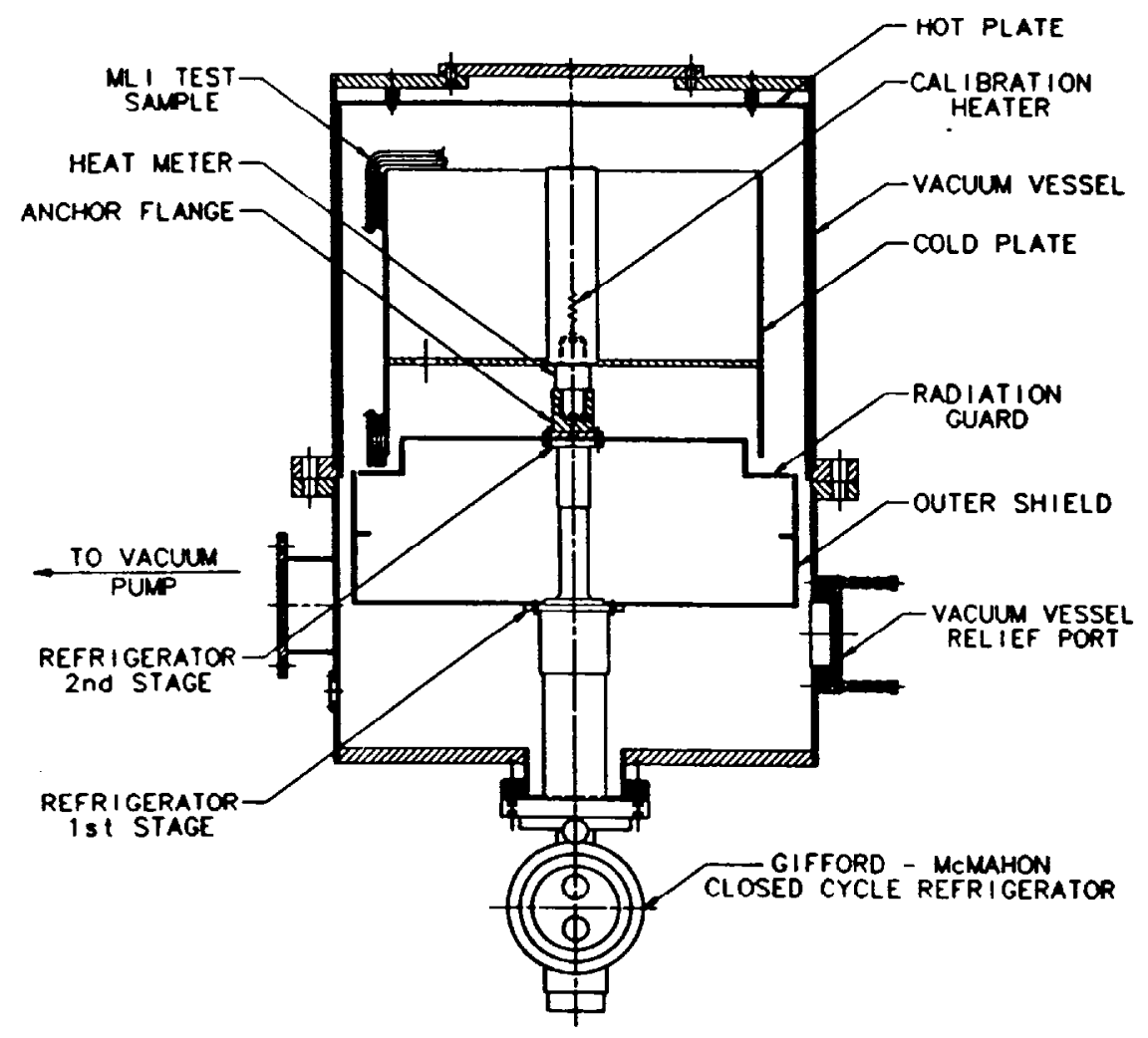

Figure 1. Modified heat leak test facility. 